\title{
Outcome of reoperative aortic root or ascending aorta replacement after prior aortic valve replacement
}

\author{
Yaojun Dun, Yi Shi, Hongwei Guo, Yanxiang Liu, Xiangyang Qian, Xiaogang Sun, Cuntao Yu \\ Department of Cardiovascular Surgery, Fuwai Hospital, National Center for Cardiovascular Diseases, Chinese Academy of Medical Sciences and \\ Peking Union Medical College, Beijing, China \\ Contributions: (I) Conception and design: Y Dun, X Sun; (II) Administrative support: X Qian, X Sun, C Yu; (III) Provision of study materials or \\ patients: Y Dun, Y Shi, H Guo; (IV) Collection and assembly of data: Y Dun, Y Liu; (V) Data analysis and interpretation: Y Dun; (VI) Manuscript \\ writing: All authors; (VII) Final approval of manuscript: All authors. \\ Correspondence to: Xiaogang Sun. 167\# Beilishi Road, Xicheng District, Beijing 100037, China. Email: xiaogangsunl@163.com.
}

\begin{abstract}
Background: There are limited data regarding the clinical outcomes of reoperative aortic root or ascending aorta replacement after prior aortic valve replacement (AVR). We aimed to analyze outcomes of reoperative aortic root or ascending aorta replacement after prior AVR.

Methods: Eighty patients with prior AVR underwent reoperative aortic root or ascending aorta replacement in our hospital. The indications were root or ascending aortic aneurysm in 36 patients, root or ascending aortic dissection in 37 , root false aneurysm in 2, prosthesis valve endocarditis (PVE) with root abscess in 2, Behçet's disease (BD) with root destruction in 3 patients. An elective surgery was performed in 63 patients and an emergent surgery in 17. The survival and freedom from aortic events during the followup were evaluated with the Kaplan-Meier survival curve and the log-rank test.

Results: The operative techniques included ascending aorta replacement in 14 patients, ascending aorta replacement with AVR in 3, prosthesis-sparing root replacement (PSRR) in 35, Bentall procedure in 24, and Cabrol procedure in 4 patients. Operative mortality was $1.3 \%$ (1/80). A composite of adverse events occurred in 5 patients, including 1 operative death, 2 stroke and 3 renal failure necessitating hemodialysis. The mean follow-up was $35.5 \pm 22.1$ months. Five late deaths occurred. The Kaplan-Meier survival at 1 year, 3 years and 6 years were $97.5 \%, 91.1 \%$ and $84.1 \%$, respectively. Aortic events developed in 3 patients. The freedom from aortic events at 1-year, 3-year, and 6-year were $100 \%, 96.3 \%$ and $88.9 \%$, respectively. There were no differences in survival and freedom from aortic events between the elective group and the emergent group.

Conclusions: Reoperative aortic root or ascending aorta replacement after prior AVR could be performed to treat the root or ascending pathologies after AVR, with satisfactory early and midterm outcomes.
\end{abstract}

Keywords: Aortic root; ascending aorta; reoperation; aortic valve replacement (AVR)

Submitted Oct 15, 2020. Accepted for publication Dec 17, 2020.

doi: $10.21037 /$ jtd-20-3081

View this article at: http://dx.doi.org/10.21037/jtd-20-3081

\section{Introduction}

Primary aortic root or ascending aorta replacement currently represents low-risk operative procedure, especially when performed electively, and the long-term outcomes are very optimal $(1,2)$. As the number of cardiac procedures accumulates and the patient population ages, it is not surprising that many patients will require redo operations on the aortic root or ascending aorta. However, reoperative root or ascending replacement after previous cardiac procedures is not only technically challenging, but also associated with increased mortality and morbidity when compared with primary root or ascending replacement (3).

Aortic valve replacement (AVR) is a very safe and reproducible procedure in the current era. But it is associated with some late complications, including prosthetic 
valve dysfunction, prosthesis valve endocarditis (PVE), and paravalvular leak (PVL) (4-6). Patients who develop these complications and patients who develop proximal aortic dissection or aneurysm after AVR may require reoperative root or ascending replacement. However, the aortic annulus structure in patients with prior AVR is much different with normal aortic annulus. And AVR could change valvular hemodynamics and impact aortic wall stress distribution, which result in different root and ascending structure (7). The root and ascending structure could also be influenced by aortic incision during AVR. Therefore, we think that reoperative root or ascending replacement after prior AVR represents a distinctive and challenging subgroup. However, there are limited data regarding the clinical outcomes of this subgroup. With the accumulation of experiences in reoperative cardiac surgery, and increasing number of reoperative root or ascending replacement after prior AVR, it has become necessary to redefine the risk and outcomes of this cohort. The aim of the present study was to investigate the early and midterm outcomes of reoperative root or ascending replacement after prior AVR. We present the following article in accordance with the STROBE reporting checklist (available at http://dx.doi.org/10.21037/jtd-20-3081).

\section{Methods}

\section{Patient population}

The study was conducted in accordance with the Declaration of Helsinki (as revised in 2013). The study was approved by the institutional review board of Fuwai Hospital. A waiver of informed consent was granted due to the design of the study. The Fuwai Aortic Surgery database was retrospectively reviewed from June 2013 to September 2019. Patients who underwent reoperative aortic root or ascending aorta replacement after prior AVR were included in our study. And patients who had underwent prior open aortic surgery were excluded. Finally, we enrolled 80 patients. We compared in-hospital and midterm follow-up data between 63 patients undergoing elective surgery (group 1 ) and 17 patients undergoing emergent surgery (group 2). The emergent surgery was defined as surgery within 3 days from admission. The interval from admission to surgery was $11.1 \pm 7.2$ days in group 1 and $0.6 \pm 1.1$ days in group $2(\mathrm{P}<0.001)$.

The mean age was $56.4 \pm 13.1$ years, and 55 patients $(68.8 \%)$ were male. The Euroscore was $7.0 \pm 1.2$ in group 1 and $11.9 \pm 0.9$ in group $2(\mathrm{P}<0.001)$. Thirty-nine patients (48.8\%) received prior AVR in our hospital, whereas 41 patients $(51.2 \%)$ in outside hospital. Fourteen patients $(17.5 \%)$ received prior AVR with bioprosthesis, while the remaining 66 patients $(82.5 \%)$ with mechanical prosthesis. Patients characteristics are outlined in Table 1.

\section{Indications for reoperations}

The indications for reoperations included root or ascending aortic aneurysm in 36 patients, root or ascending aortic dissection in 37, root false aneurysm in 2, PVE with root abscess in 2, Behçet's disease (BD) with root destruction in 3 patients (Figure 1). The mean interval between the prior AVR and the current reoperation were $8.3 \pm 5.7$ years (range, 4 months -24 years).

Prosthetic valve dysfunction is defined as moderate or severe prosthetic stenosis (mean aortic gradient $\geq 20 \mathrm{mmHg}$ ), and/or moderate or severe prosthetic regurgitation. In this cohort, 22 patients presented with prosthetic valve dysfunction, including 6 with aortic dissection, 15 with aortic aneurysm, and 1 with root false aneurysm. And 13 patients presented with moderate or severe PVL, including 1 with aortic dissection, 6 with aortic aneurysm, 1 with root false aneurysm, 2 with PVE, and 3 with BD. The details are listed in Table 1.

\section{Operative strategy}

Computed tomography (CT) was preoperatively performed in all patients to identify root or ascending pathologies. The CT scans of patients with different indications are shown in Figure 2. CT could also demonstrate the relationships between the posterior aspect of the sternum and the aorta, the heart, and bypass grafts. If the preoperative CT scan demonstrated minimal space between the posterior aspect of the sternum and the heart, the femoral artery and vein were exposed before sternotomy.

All patients underwent reoperation through a redo sternotomy. After the skin incision and removal of the sternal wires, the sternum was opened with the oscillating saw. The adhesions surrounding the aorta and the right atria were carefully dissected. If the left mammary artery was used for a previous coronary artery bypass grafting (CABG), the mammary artery was mobilized enough to place a padded bulldog clamp during the aortic-clamp period. Arterial cannulation was distributed in the following fashion: aortic, $n=2$; femoral, $n=72$; axillary, $n=6$. The right atrium or both venae cava were used for venous cannulation, then the cardiopulmonary bypass $(\mathrm{CPB})$ was initiated. 
Table 1 Baseline characteristics by study group ${ }^{\mathrm{a}}$

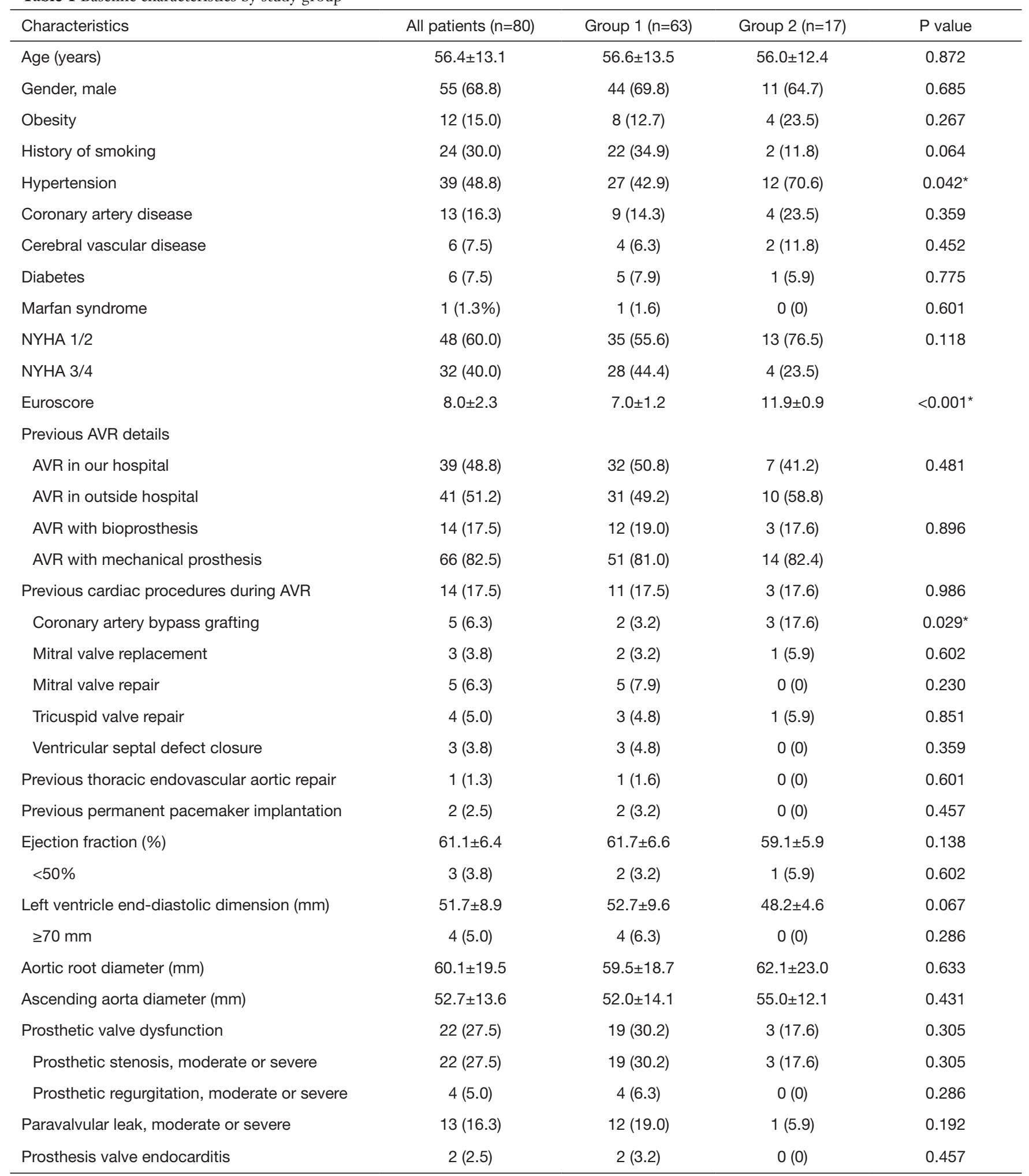

Categorical data are presented as $\mathrm{n}(\%)$, and continuous data as the mean \pm standard deviation. ${ }^{\mathrm{a}}$ Group 1 , elective surgery; group 2 , emergent surgery. ${ }^{*} \mathrm{P}$ values indicate statistical significance $(\mathrm{P}<0.05)$. AVR, aortic valve replacement; NYHA, New York Heart Association. 


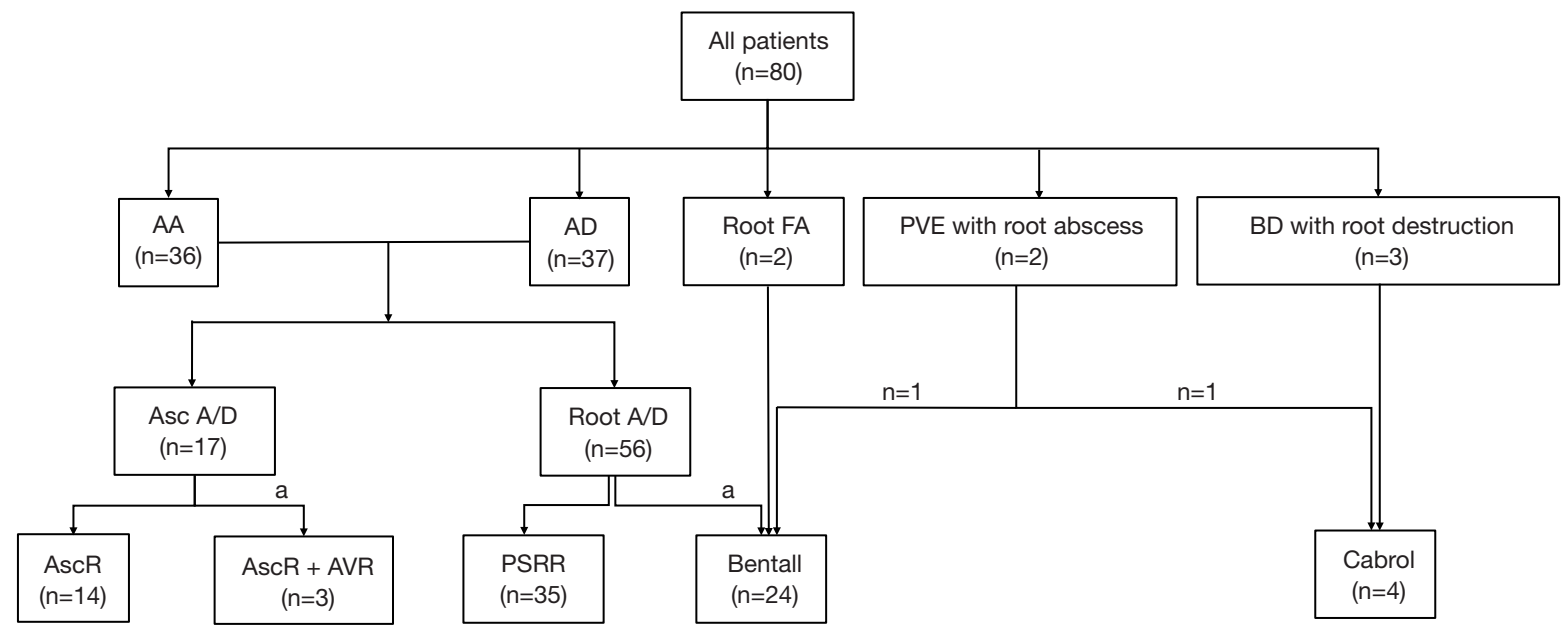

Figure 1 Surgical indications and techniques of reoperations. AA, aortic aneurysm; AD, aortic dissection; Root FA, root false aneurysm; PVE, prosthesis valve endocarditis; BD, Behçet's disease; Asc A/D, ascending aneurysm or dissection; Root A/D, root aneurysm or dissection; AscR, ascending replacement; AVR, aortic valve replacement; PSRR, prosthesis-sparing root replacement; a, prosthetic valve dysfunction, or paravalvular leak.
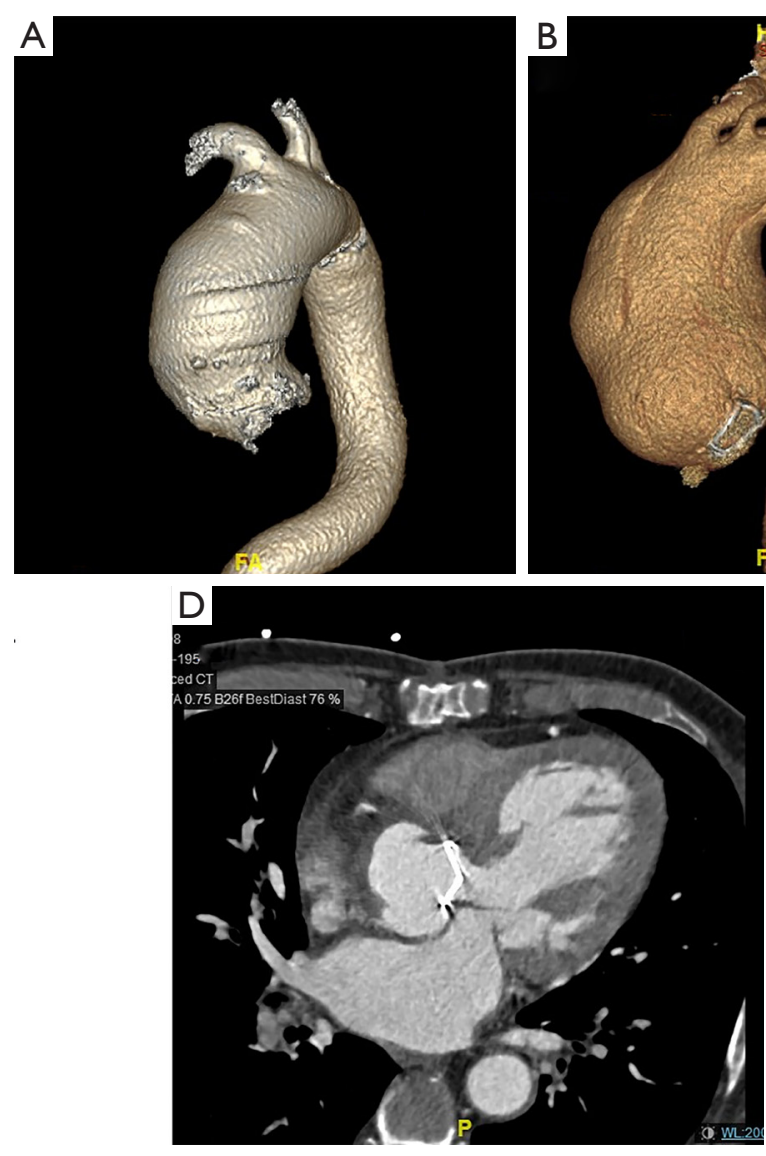

Figure 2 Computed tomographic scans of 5 patients with (A) aortic aneurysm; (B) aortic dissection; (C) root false aneurysm; (D) prosthesis valve endocarditis with root abscess; (E) Behçet's disease with root destruction.
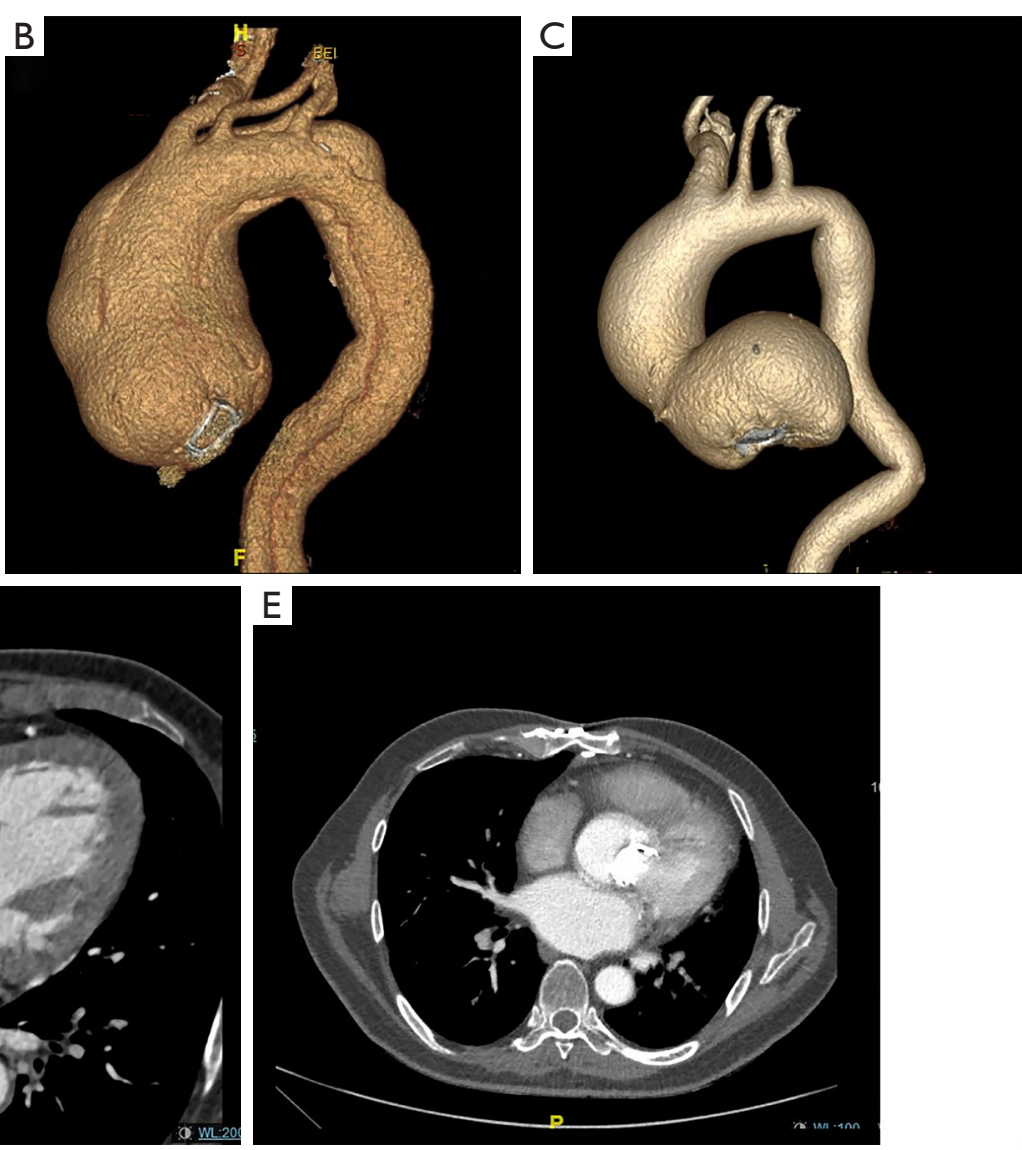
After the rectal temperature decreased to about $30{ }^{\circ} \mathrm{C}$, the ascending aorta was clamped. Then the cold blood cardioplegia was perfused through aortic root or administered into the coronary ostia under direct vision, depending on the competency of the prosthesis. After the heart was arrested, the root or ascending replacement was performed. We examined the previous prosthesis first. According to different indications, the involvement of aortic pathologies, the function of previous prosthesis, we selected different surgical techniques and performed the surgery electively or emergently.

For patients with prosthetic valve dysfunction, PVE or PVL, we would explant the prosthesis during the operation, otherwise we would preserve the prosthesis. For patients with the ascending aneurysm or dissection, ascending aorta replacement with or without additional re-AVR could be selected. For patients with the root aneurysm, dissection, or false aneurysm, Bentall procedure could be performed in patients with prosthetic valve dysfunction or PVL, otherwise a prosthesis-sparing root replacement (PSRR) could be performed (8). When performing PSRR, a tube Dacron graft was sutured to the sewing ring of the previously implanted prosthesis using interrupted pledgeted 2-0 braided polyester sutures or running 2-0 prolene suture, then the reimplantation of the coronary ostia was performed as usual. For patients with PVE or BD, Bentall procedure or Cabrol procedure could be selected. The Cabrol procedure was performed with an $8-\mathrm{mm}$ interposition graft which was anastomosed to the coronary ostia, then anastomosed to the aortic graft in a side-to-side fashion. When performing root replacement including Bentall, Cabrol and PSRR, we could select the button technique or the inclusion technique with a perigraft-to-right atria shunt, according to the root anatomy, and surgeons' experiences.

In cases of previous CABG using saphenous vein grafts, a Carrel patch (button) was created with a native aortic wall and reimplanted to the aortic Dacron graft. For patients with the arch pathologies, the arch surgery should be performed simultaneously. Hemiarch replacement, partial arch replacement, subtotal arch replacement, total arch replacement with or without frozen elephant trunk technique, and hybrid total arch repair could be selected after comprehensive consideration of the characteristics of arch pathologies, the involvement segment of the lesions, the condition of patients and the surgeons' experiences. Hybrid total arch repair was performed without hypothermia circulatory arrest (HCA), while HCA was required in all patients undergoing subtotal or total arch replacement, and most patients undergoing hemiarch or partial arch replacement. For the other patients who received hemiarch or partial arch replacement without HCA, the distal anastomosis was performed with the clamping of distal arch and the innominate artery, and the distal perfusion was achieved through the femoral cannulation. Whenever HCA was foreseen to perform open distal arch anastomosis, the rectal temperature was cooled to $22-26{ }^{\circ} \mathrm{C}$. And selective antegrade cerebral perfusion (SCP) was achieved via the innominate artery or axillary artery (except 1 patient via the left common carotid artery), with the flow of $5-10 \mathrm{~mL} / \mathrm{kg} \cdot \mathrm{min}$.

\section{Definitions, study endpoints and statistical analysis}

Obesity was defined as having a body mass index of more than 28. History of smoking was defined as continuous or cumulative smoking for more than 6 months. Operative death was defined as death within 30 days of surgery or before final discharge from hospitalization. Acute renal failure was defined as the ratio of postoperative serum creatinine to preoperative serum creatinine more than 2 , or renal failure necessitating hemodialysis. Prolonged ventilation was defined as mechanical ventilation for more than 48 hours after surgery. Aortic events were defined as recurrent aortic dissection, aortic rupture, deaths of aortic cause and aortic-related reinterventions.

The primary endpoints were a composite of adverse events, including operative death, stroke, paraplegia, and renal failure necessitating hemodialysis. The secondary endpoints were acute renal failure, and prolonged ventilation.

Continuous variables were expressed as the mean \pm standard deviation or median (range), and categoric variables expressed as number (percentage). The continuous variables between the 2 groups were compared with the Student t test or the Wilcoxon rank sum test. The categorical variables were compared using chi-square analysis. Risk factors for a composite of adverse events, acute renal failure, and prolonged ventilation were identified with univariable and multivariable logistic regression model analysis. The survival and freedom from aortic events were evaluated with the Kaplan-Meier survival curve and the log-rank test.

\section{Results}

\section{Operative data}

One patient suffered aneurysm rupture during sternotomy. 
Table 2 Indications and surgical techniques by study group ${ }^{\mathrm{a}}$

\begin{tabular}{|c|c|c|c|c|c|}
\hline Indications & All patients $(n=80)$ & \multicolumn{2}{|c|}{ Group $1(\mathrm{n}=63)$} & \multicolumn{2}{|c|}{ Group $2(n=17)$} \\
\hline \multirow[t]{3}{*}{ Aortic aneurysm } & $36(45.0)$ & PSRR & 14 & & \\
\hline & & Bentall & 16 & & \\
\hline & & AscR & 4 & & \\
\hline \multirow[t]{4}{*}{ Aortic dissection } & $37(46.3)$ & PSRR & 11 & PSRR & 9 \\
\hline & & Bentall & 4 & Bentall & 2 \\
\hline & & AscR & 6 & AscR & 4 \\
\hline & & & & Asc + AVR & 1 \\
\hline PVE + root abscess & & Cabrol & 1 & & \\
\hline $\mathrm{BD}+$ root destruction & $3(3.8)$ & Cabrol & 3 & & \\
\hline
\end{tabular}

Data are presented as $\mathrm{n}(\%)$ or as indicated. ${ }^{2}$ Group 1, elective surgery; group 2, emergent surgery. AscR, ascending aorta replacement; AVR, aortic valve replacement; BD, Behçet's disease; No., number; PSRR, prosthesis-sparing root replacement; PVE, prosthesis valve endocarditis.

Then deep HCA with systemic cooling to $18^{\circ} \mathrm{C}$ through femoral cannulation was emergently applied, followed by resternotomy and reoperative aortic surgery. In addition, 2 patients received revisions after aortic unclamping. One patient received mitral valve repair for new-onset mitral regurgitation. The other patient received PSRR for root false aneurysm at first. But the massive bleeding of aortic root developed. Then we removed the sutures and previous prosthesis, and performed an Bentall procedure with biological valved conduit.

The previous prosthesis was explanted in 31 patients and preserved in 49 patients. The operations included ascending aorta replacement in 14 patients, ascending aorta replacement with AVR in 3, PSRR in 35, Bentall procedure in 24, and Cabrol procedure in 4 patients (Figure 1; Table 2). Fourteen mechanical valved conduits and 10 biological valved conduits were used in patients undergoing Bentall procedures. For 1 patient with PVE, Bentall procedure was performed with a valved homograft. In the other 1 patient with PVE and 3 patients with BD, the coronary ostia were friable because of serious infection or inflammatory lesions, and Cabrol procedure with mechanical valved conduit was performed. Among 63 patients receiving root replacement, the button technique was used in only 6 patients ( 3 Bentall procedures and 3 PSRR).
Thirty-two patients in group 1 and 16 patients in group 2 underwent additional procedures $(50.8 \%$ vs. $94.1 \%$, $\mathrm{P}=0.001)$. The mean $\mathrm{CPB}$ time, aortic-clamp time was $158.4 \pm 63.5$ and $100.4 \pm 39.9 \mathrm{~min}$, respectively. HCA was required in 19 patients undergoing elective surgery and 10 patients undergoing emergent surgery $(30.2 \%$ vs. $58.8 \%$, $\mathrm{P}=0.029)$. The mean SCP duration was $17.2 \pm 5.6 \mathrm{~min}$. The operative details are listed in Table 3.

\section{Operative mortality and morbidity}

Operative mortality was $1.3 \%(1 / 80)$. The patient was a 63-year-old male. He received PSRR at first, and turned to Bentall procedure for massive root bleeding, as previously described. The postoperative course was uneventful, and he discharged 13 days after surgery. However, he suffered sudden death for unknown reason 7 days after discharge.

Primary endpoints occurred in 5 patients, including 1 operative death, 2 stroke and 3 renal failure necessitating hemodialysis. Fifteen patients suffered acute renal failure, and 23 patients had prolonged ventilation. In addition, 3 patients received reoperation for bleeding, 2 patients received tracheostomy, 2 patients had hepatic dysfunction. And atrioventricular block requiring permanent pacemaker implantation during the same hospitalization occurred in 1 
Table 3 Operative and postoperative results by study group ${ }^{a}$

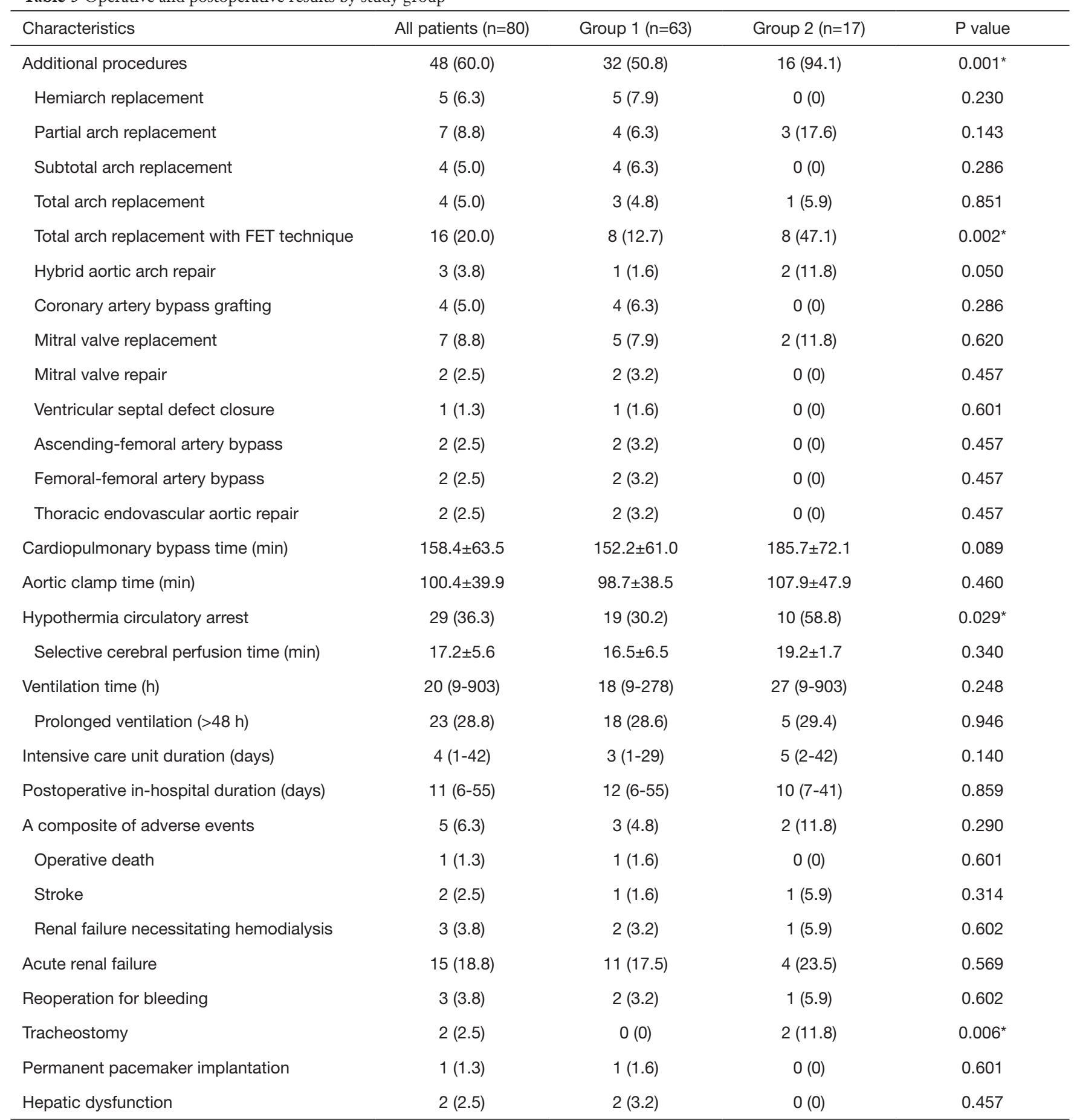

Categorical data are presented as $\mathrm{n}(\%)$, and continuous data as the mean \pm standard deviation or median (range). ${ }^{2} \mathrm{Group} 1$, elective surgery; group 2, emergent surgery. ${ }^{*} \mathrm{P}$ values indicate statistical significance $(\mathrm{P}<0.05)$. FET, frozen elephant trunk. 
Table 4 Univariable and multivariable regression analysis for acute renal failure and prolonged ventilation

\begin{tabular}{|c|c|c|c|c|}
\hline Study endpoints & \multicolumn{2}{|c|}{ Univariable analysis } & \multicolumn{2}{|c|}{ Multivariable analysis } \\
\hline \multicolumn{5}{|l|}{ Acute renal failure } \\
\hline Emergent surgery & $1.46(0.40-5.32)$ & 0.571 & $1.01(0.23-4.38)$ & 0.993 \\
\hline Age $\geq 65$ years old & $0.26(0.06-1.27)$ & 0.096 & $0.37(0.07-1.90)$ & 0.234 \\
\hline Hypothermic circulatory arrest & $4.84(1.46-16.06)$ & $0.01^{*}$ & $3.89(1.05-14.39)$ & $0.042^{*}$ \\
\hline \multicolumn{5}{|l|}{ Prolonged ventilation } \\
\hline Emergent surgery & $1.04(0.32-3.38)$ & 0.946 & $1.06(0.29-3.85)$ & 0.926 \\
\hline Age $\geq 65$ years old & $1.51(0.55-4.16)$ & 0.423 & $1.88(0.62-5.71)$ & 0.264 \\
\hline CPB time $\geq 200 \mathrm{~min}$ & $3.13(0.95-10.27)$ & 0.06 & $3.73(1.03-13.50)$ & $0.045^{\star}$ \\
\hline Hypothermic circulatory arrest & $1.19(0.44-3.23)$ & 0.734 & $0.99(0.30-3.22)$ & 0.988 \\
\hline
\end{tabular}

${ }^{*} \mathrm{P}$ values indicate statistical significance $(\mathrm{P}<0.05)$. CPB, cardiopulmonary bypass; OR $(95 \% \mathrm{Cl})$, odds ratio $(95 \%$ confidence interval).

patient. The postoperative results are listed in Table 3.

All patients were eventually discharged from hospital.

Two patients were transferred to another hospital after discharge, for further treatment of respiratory failure. And there were 3 unplanned readmissions within 1 month after discharge. The causes of readmissions were poor healing of chest wound, poor healing of groin wound, and massive pericardial effusion, respectively.

No risk factor was associated with a composite of adverse events using univariable and multivariable regression analysis. On univariable regression analysis, HCA was identified to be a risk factor for acute renal failure $(\mathrm{P}=0.01)$, and no risk factor was related to prolonged ventilation. On multivariable regression analysis, HCA was an independent risk factor of acute renal failure $(\mathrm{P}=0.042)$, and $\mathrm{CPB}$ time of more than 200 minutes was an independent risk factor of prolonged ventilation $(\mathrm{P}=0.045)$. The results of univariable and multivariable regression analysis for acute renal failure and prolonged ventilation are shown in Table 4.

\section{Follow-up}

The follow-up data were available for all 79 survivors. The mean follow-up time was $35.5 \pm 22.1$ months (range: 1-77 months). Five late deaths occurred during the follow-up. Two deaths were due to heart failure, and the death causes for the other 3 patients were ruptured aortic dissection, cerebral hemorrhage, and severe pneumonia, respectively. The Kaplan-Meier survival at 1 year, 3 years and 6 years were $97.5 \%, 91.1 \%$ and $84.1 \%$, respectively.

Aortic events occurred in 3 patients. One patient died of ruptured aortic dissection 65 months after surgery; 1 patient underwent thoracoabdominal aortic aneurysm repair 20 months after surgery, for dilation of the false lumen in the thoracoabdominal aorta; and 1 patient received percutaneous aorta-right atria fistula closure 17 months later. The freedom from aortic events at 1-year, 3-year, and 6 -year were $100 \%, 96.3 \%$ and $88.9 \%$, respectively.

Survival at 6 years in patients who underwent elective surgery (group 1) and emergent surgery (group 2) was $81.9 \%$ and $100 \%$, respectively (log-rank $\mathrm{P}=0.28$; Figure $3 A$ ), and freedom from aortic events at 6 years in the 2 groups was $89.6 \%$ and $88.9 \%$, respectively (log-rank $\mathrm{P}=0.29$; Figure $3 B$ ).

In addition, 1 patient required permanent hemodialysis after discharge; 2 patients suffered stroke; and 1 patient received permanent pacemaker implantation. One patient with coronary artery disease underwent 3 percutaneous coronary interventions for left main artery lesions.

\section{Discussion}

Although the real incidence of reoperative root or 
A

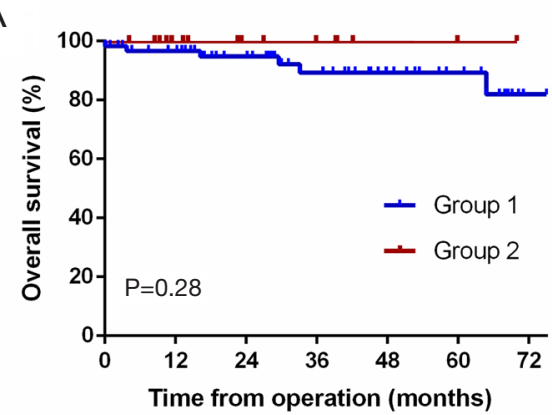

Patients at risk

$\begin{array}{cccccccc}\text { Group 1 } & 63 & 56 & 45 & 33 & 21 & 15 & 5 \\ \text { Group 2 } & 17 & 12 & 8 & 6 & 3 & 2 & 1\end{array}$

B

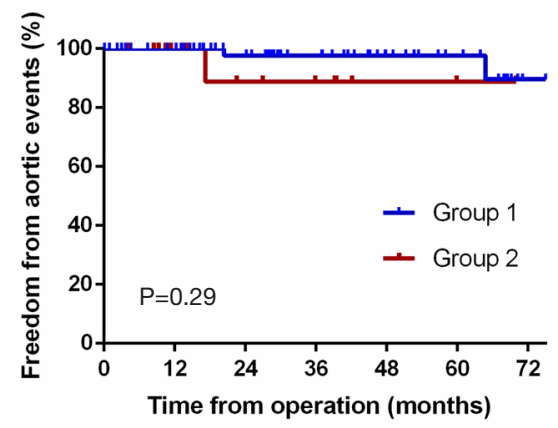

Patients at risk

$\begin{array}{cccccccc}\text { Group 1 } & 63 & 56 & 44 & 33 & 21 & 15 & 5 \\ \text { Group 2 } & 17 & 12 & 8 & 6 & 3 & 2 & 1\end{array}$

Figure 3 The Kaplan-Meier curves show (A) overall survival comparing group 1 (elective surgery) and group 2 (emergent surgery), (B) freedom from aortic events comparing group 1 and group 2.

ascending replacement is unknown, it is anticipated that more and more patients will require reoperations on the proximal aorta. Among the types of primary procedures in patients undergoing reoperations on the proximal aorta, isolated AVR was the most common type, ranged from $28.6 \%$ to $65.5 \%$ (9-11). However, reports that specifically focused on reoperative root or ascending replacement after AVR are very limited. In present study, we summarized our experiences of reoperative root or ascending replacement after AVR and analyzed the outcomes of these patients. Our study has demonstrated that these complex reoperations on the proximal aorta can be performed safely in patients with prior AVR at a high-volume aortic center.

As described above, the indications for reoperations in this study included aortic aneurysm, aortic dissection, root false aneurysm, PVE with root abscess, and BD with root destruction. It is important to optimize the surgical strategy according to different indications, the function of previous prosthesis, and the involvement of aortic pathologies.

The aortic dissection was the most common indication in this cohort, accounted for $46.3 \%(37 / 80)$. Type I aortic dissection develops in $0.2-2.3 \%$ of patients after AVR (12). In a retrospective study enrolling 13,205 adults, Itagaki and colleagues found that the long-term incidence of thoracic aortic dissection after AVR was significantly higher in patients with Marfan syndrome $(5.5 \pm 2.7 \%)$ compared with those with bicuspid valves $(0.55 \pm 0.21 \%)$ and patients with acquired aortic valve disease $(0.41 \pm 0.08 \%$, $\mathrm{P}<0.001)(13)$. And Tsutsumi also identified that aortic regurgitation combined with hypertension, male, and thinned or fragile aortic walls in patients with ascending dilatation ( $\geq 45 \mathrm{~mm}$ diameter) at the time of AVR may be risk factors for late aortic dissection (12). Aortic aneurysm was the second most common $(45.0 \%, 36 / 80)$ indication in this study. Due to progressive aortic dilatation, root or ascending aneurysm was also a common aortopathy after AVR (14). Itagaki also discovered that thoracic aortic aneurysms after AVR in late follow-up were significantly more likely to be diagnosed in patients with Marfan syndrome $(10.8 \pm 4.4 \%)$ compared with those with bicuspid valves $(4.8 \pm 0.8 \%)$ and control group patients $(1.4 \pm 0.2 \%)$ $(\mathrm{P}<0.001)$ (13). In this cohort, many patients already had root or ascending dilatation $(45-55 \mathrm{~mm})$ at the time of AVR, but they did not receive aortic surgery due to high operative risk in some remote hospitals. It has been widely accepted that the patients with proximal aortic dissection or aneurysm after AVR had to receive reoperative root or ascending replacement. Patients could also develop root false aneurysm after prior AVR, due to the fragility of aortic wall. And reoperative root replacement was the only alternative for this pathology. In this cohort, we performed root replacement for 2 patients with root false aneurysm.

Prosthetic valve dysfunction was not an uncommon complication after AVR, and the causes included structure valve deterioration of bioprosthesis, valve thromboembolism, tissue ingrowth, subvalvular pannus formation. PVL was also a common complication, with a reported incidence of $2-17 \%$ after surgical AVR (15). PVL is due to incomplete apposition of the sewing ring to the native tissue, and it occurs as a result of a variety of factors, including infection, suture dehiscence, calcified annulus, friability of the annulus tissue, lack of space to locate the valve sutures and technical difficulties in accessing the ring valve tissue (16). Isolated prosthetic valve dysfunction or 
PVL after AVR could be treated by re-AVR or transcatheter closure of PVL (16). But patients with concurrent root pathologies had to receive reoperative root replacement. In this study, 21 patients with prosthetic valve dysfunction or PVL had concurrent root aneurysm or dissection, and they underwent Bentall procedure. Specially, PVL was caused by PVE in 2 patients, BD in 3 patients. They had severe root abscess or destruction, and root replacement was also mandatory. For patients with severe infection, a cryopreserved valved homograft should be used to replace the root to decrease the risk of recurrent infection (17). And we used valved homograft in 1 patient with PVE. Indeed, PVE was also the prevailing indications for reoperative root replacement in some previous studies $(9,18)$. BD with severe aortic regurgitation could be primarily managed by isolated AVR (19). However, many patients would develop PVL after AVR, due to prosthesis dehiscence (19). And the surgical treatment of PVL in patients with BD was intractable. When severe root destruction was encountered, reoperative aortic root replacement was the most suitable solution (20).

Removal of the previous prosthesis requires operating on a sometimes weakened and pathological aortic annulus, which would injure the adjacent structures (8). And distortion of the aortic annulus after the removal of the prosthesis may impel the surgeon to implant a new smaller prosthesis (8). Therefore, we thought that a prosthesis without prosthetic valve dysfunction or PVL should be spared whenever possible when performing aortic root replacement. In our study, most patients (43.8\%) received PSRR.

Although various technical improvements have been achieved, reoperative root or ascending replacement after previous cardiac procedures still remains a surgical challenge, with the mortality of $5.4 \%$ to $17.9 \%(3,9-$ $11,17,18,21,22)$. Only few studies calculated the mortality of reoperative root replacement in patients with prior AVR [Szeto et al. 14.1\% (18) and Esaki et al. 14.1\% (22)]. In our study, the mortality was only $1.3 \%$, much less than that of previous reports. The optimal results could be partly explained by young age, less preoperative comorbidities, and overall good left heart function (only 3 patients with ejection fraction $<50 \%$ ) in this cohort. As the most famous cardiovascular center in China, we implemented strict operation grading system. Only very experienced senior surgeons were eligible for redo aortic surgeries, which also resulted in low mortality in this study. According to previous studies, the possible risk factors of operative death included older age, preoperative renal failure or lung disease, prior myocardial infarction, preoperative functional class III/IV, prolonged CPB duration $(17,18,22)$. Although HCA could reduce the metabolic rate of the tissue, it was also associated with endothelial dysfunction in renal arteries, and it was a strong independent risk factor for postoperative renal impairment (23). Due to exposure of blood to abnormal surfaces, CPB could result in systemic inflammatory responses, which was detrimental to many organs including lungs. Nadeem had identified that prolonged CPB duration was associated with prolonged ventilation (24). According to multivariable regression analysis in this study, we confirmed that HCA was related to acute renal failure $(\mathrm{P}=0.042)$, and $\mathrm{CPB}$ time of more than 200 minutes was related to prolonged ventilation $(\mathrm{P}=0.045)$.

In addition, due to the anatomic proximity of conduction tissue, complete atrioventricular block was not an uncommon complication after reoperations on the proximal aorta, which was ranged from $4.1 \%$ to $17.3 \%$ $(9,10,17,18)$. In our study, only 1 patient $(1.3 \%)$ developed complete atrioventricular block requiring pacemaker implantation. The main reason was higher proportion of PSRR (43.8\%) and ascending replacement (17.5\%) in this cohort. The Dacron graft was anastomosed to the sewing ring of the previous prosthesis or sinotubular junction when performing the two techniques, which was impossible to injure the conduction tissue. In addition, patients in this cohort had less root adhesions than patients with previous root surgery. Therefore, the trauma of root at reoperation could be reduced, which could also result in lower incidence of complete atrioventricular block.

The midterm outcome was also satisfactory in our study, with 6-year survival of $84.1 \%$ and freedom from aortic events of $88.9 \%$. Other series have reported 5 -year survival ranging from $72.6 \%$ to $83.0 \%(9,18,22)$. We found that emergent surgery did not impact overall survival and freedom from aortic events. According to previous studies, the survival was impacted by patient age, previous CABG, heart function class and preoperative chronic kidney disease $(11,18,22)$.

During the follow-up, 5 late deaths and 3 aortic events occurred. All 3 patients with aortic events had surgical indication of aortic dissection. Indeed, for many patients with aortic dissection, the false lumen of the distal aorta could be patent after surgery. Progressive dilation of the distal aorta was prone to develop in these patients, and they may experience ruptured dissection of the distal aorta or require open thoracoabdominal reoperations. In this study, 
1 patient received thoracoabdominal aortic aneurysm repair due to progressive dilatation of an untreated dissected aorta, and 1 patient experienced ruptured dissection of the distal aorta. In addition, 1 patient received percutaneous aortaright atria fistula closure during the follow-up. This patient underwent reoperative root replacement using the inclusion technique with a perigraft-to-right atria shunt. However, this shunt did not close and turn into the persistent aortaright atria fistula which require reintervention. The inclusion technique with a perigraft-to-right atria shunt was to create the connection between the perigraft and the right atria by direct anastomosis. This procedure could control the surgical bleeding and improve hemostasis of aortic root and coronary anastomosis. Therefore, we thought that it was especially suitable for reoperations of aortic root. But it was also associated with some late complications, including persistent aorta-right atria fistula, root false aneurysm, coronary artery-to-perigraft fistula, right heart failure because of persistent left-to-right shunt (25). And we still used this technique in most patients undergoing reoperations on root.

There are some inevitable limitations to this study. Firstly, due to the low incidence of operative death, and complications, this study is not powered for evaluating the risk factors of the first endpoint listed in the paper. Only risks factor of acute renal failure and prolonged ventilation were analyzed. Till now, no study specially reported the outcome of reoperative root or ascending replacement after prior AVR. Therefore, we could not compare our findings carefully with the previous studies. Additionally, the patient population is very heterogenous, which makes it difficult to conclude on the outcome of the procedures.

\section{Conclusions}

In conclusion, reoperative aortic root or ascending aorta replacement after prior AVR could be indicated for aortic aneurysm, aortic dissection, root false aneurysm, PVE with root abscess, and BD with root destruction. Although technically complex and challenging, reoperative aortic root or ascending aorta replacement could be performed safely with satisfactory early and midterm outcomes.

\section{Acknowledgments}

Funding: This work was supported by Beijing Municipal Science and Technology Commission Capital characteristic clinic project (Z181100001718197).

\section{Footnote}

Reporting Checklist: The authors have completed the STROBE reporting checklist. Available at http://dx.doi. org/10.21037/jtd-20-3081

Data Sharing Statement: Available at http://dx.doi. org/10.21037/jtd-20-3081

Conflicts of Interest: All authors have completed the ICMJE uniform disclosure form (available at http://dx.doi. org/10.21037/jtd-20-3081). The authors have no conflicts of interest to declare.

Ethical Statement: The authors are accountable for all aspects of the work in ensuring that questions related to the accuracy or integrity of any part of the work are appropriately investigated and resolved. The study was conducted in accordance with the Declaration of Helsinki (as revised in 2013). The study was approved by the Institutional Ethics Committee of Fuwai Hospital, and individual consent for this retrospective analysis was waived.

Open Access Statement: This is an Open Access article distributed in accordance with the Creative Commons Attribution-NonCommercial-NoDerivs 4.0 International License (CC BY-NC-ND 4.0), which permits the noncommercial replication and distribution of the article with the strict proviso that no changes or edits are made and the original work is properly cited (including links to both the formal publication through the relevant DOI and the license). See: https://creativecommons.org/licenses/by-nc-nd/4.0/.

\section{References}

1. Preventza O, Coselli JS, Price MD, et al. Elective primary aortic root replacement with and without hemiarch repair in patients with no previous cardiac surgery. J Thorac Cardiovasc Surg 2017;153:1402-8.

2. Svensson LG, Kouchoukos NT, Miller DC, et al. Expert consensus document on the treatment of descending thoracic aortic disease using endovascular stent-grafts. Ann Thorac Surg 2008;85:S1-41.

3. Sandhu HK, Tanaka A, Zaidi ST, et al. Impact of redo sternotomy on proximal aortic repair: Does previous aortic repair affect outcomes? J Thorac Cardiovasc Surg 2020;159:1683-91.

4. McClure RS, Narayanasamy N, Wiegerinck E, et al. 
Late Outcomes for Aortic Valve Replacement With the Carpentier-Edwards Pericardial Bioprosthesis: Up to 17-Year Follow-Up in 1,000 Patients. Ann Thorac Surg 2010;89:1410-6.

5. Glaser N, Jackson V, Holzmann MJ, et al. Prosthetic Valve Endocarditis After Surgical Aortic Valve Replacement. Circulation 2017;136:329-31.

6. Kliger C, Eiros R, Isasti G, et al. Review of surgical prosthetic paravalvular leaks: diagnosis and catheter-based closure. Eur Heart J 2013;34:638.

7. Regeer MV, Versteegh MIM, Klautz RJM, et al. Effect of Aortic Valve Replacement on Aortic Root Dilatation Rate in Patients With Bicuspid and Tricuspid Aortic Valves. Ann Thorac Surg 2016;102:1981-7.

8. Luciani N, Anselmi A, de Geest R, et al. Facilitated aortic root substitution after aortic valve replacement: Technique and results of the prosthesis-sparing operation. J Thorac Cardiovasc Surg 2010;139:785-7.

9. Schepens MAAM, Dossche KM, Morshuis WJ. Reoperations on the ascending aorta and aortic root: Pitfalls and results in 134 patients. Ann Thorac Surg 1999;68:1676-80.

10. Girardi LN, Krieger KH, Mack CA, et al. Reoperations on the ascending aorta and aortic root in patients with previous cardiac surgery. Ann Thorac Surg 2006;82:1407-12.

11. Silva J, Maroto LC, Carnero M, et al. Ascending Aorta and Aortic Root Reoperations: Are Outcomes Worse Than First Time Surgery? Ann Thorac Surg 2010;90:555-60.

12. Tsutsumi K, Inoue Y, Hashizume K, et al. Risk factor analysis for acute type $\mathrm{A}$ aortic dissection after aortic valve replacement. Gen Thorac Cardiovasc Surg 2010;58:601-5.

13. Itagaki S, Chikwe JP, Chiang YP, et al. Long-Term Risk for Aortic Complications After Aortic Valve Replacement in Patients With Bicuspid Aortic Valve Versus Marfan Syndrome. J Am Coll Cardiol 2015;65:2363-9.

14. Girdauskas E, Disha K, Borger MA, et al. Long-term prognosis of ascending aortic aneurysm after aortic valve replacement for bicuspid versus tricuspid aortic valve

Cite this article as: Dun Y, Shi Y, Guo H, Liu Y, Qian X, Sun $\mathrm{X}, \mathrm{Yu} \mathrm{C}$. Outcome of reoperative aortic root or ascending aorta replacement after prior aortic valve replacement. J Thorac Dis 2021;13(3):1531-1542. doi: 10.21037/jtd-20-3081 stenosis. J Thorac Cardiovasc Surg 2014;147:276-82.

15. Duncan BF, McCarthy PM, Kruse J, et al. Paravalvular regurgitation after conventional aortic and mitral valve replacement: A benchmark for alternative approaches. J Thorac Cardiovasc Surg 2015;150:860-8.e1.

16. García E, Sandoval J, Unzue L, et al. Paravalvular leaks: mechanisms, diagnosis and management. Eurointervention 2012;8:Q41-52.

17. Luciani N, De Geest R, Anselmi A, et al. Results of Reoperation on the Aortic Root and the Ascending Aorta. Ann Thorac Surg 2011;92:898-903.

18. Szeto WY, Bavaria JE, Bowen FW, et al. Reoperative aortic root replacement in patients with previous aortic surgery. Ann Thorac Surg 2007;84:1592-8.

19. Jeong DS, Kim KH, Kim JS, et al. Long-Term Experience of Surgical Treatment for Aortic Regurgitation Attributable to Behcet's Disease. Ann Thorac Surg 2009;87:1775-82.

20. Ghang B, Kim JB, Jung SH, et al. Surgical Outcomes in Behcet's Disease Patients With Severe Aortic Regurgitation. Ann Thorac Surg 2019;107:1188-94.

21. Kirsch EW, Radu NC, Mekontso-Dessap A, et al. Aortic root replacement after previous surgical intervention on the aortic valve, aortic root, or ascending aorta. J Thorac Cardiovasc Surg 2006;131:601-8.

22. Esaki J, Leshnower BG, Binongo JN, et al. Reoperative aortic root replacement: Outcome in a contemporary series. J Thorac Cardiovasc Surg 2017;154:800-8 e3.

23. Haverich A, Hagl C. Organ protection during hypothermic circulatory arrest. J Thorac Cardiovasc Surg 2003;125:460-2.

24. Nadeem R, Agarwal S, Jawed S, et al. Impact of Cardiopulmonary Bypass Time on Postoperative Duration of Mechanical Ventilation in Patients Undergoing Cardiovascular Surgeries: A Systemic Review and Regression of Metadata. Cureus 2019;11:e6088.

25. Shi Y, Dun Y, Guo H, et al. Clinical features and surgical outcomes of type A intramural hematoma. J Thorac Dis 2020;12:3964-75. 